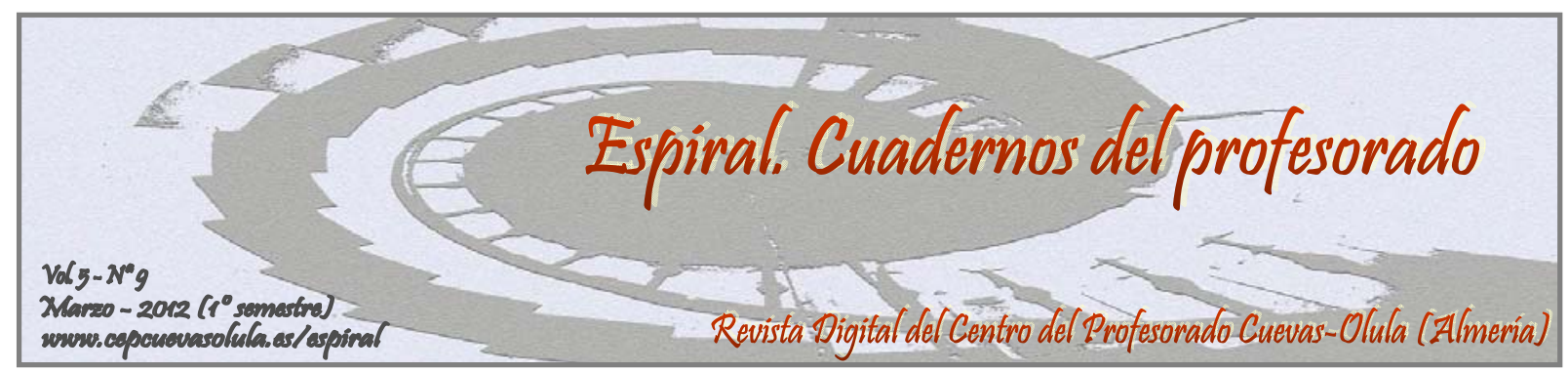

\title{
EL LENGUAJE SEXISTA EN LOS DOCUMENTOS DE PLANIFICACIÓN DE LOS CENTROS EDUCATIVOS
}

\author{
SEXIST LANGUAGE PLANNING IN THE DOCUMENTS OF SCHOOLS
}

\author{
María Estela Vargas Muñoz
}

IES Pablo Ruiz Picasso, El Ejido, Almería, España

\begin{abstract}
RESUMEN: Existen una serie de mecanismos lingüísticos que contribuyen a transmitir la impresión de que el hombre es el centro de la creatividad excluyendo a la mujer. Entre ellos está el uso del masculino como si fuera genérico, invisibilizando a las mujeres. Los centros educativos deben tener presente este aspecto a la hora de desarrollar sus documentos de planificación con el fin de que, tanto niñas como niños, se reflejen en el día a día de los colegios. En este estudio, se han seleccionado dos centros educativos de la provincia de Almería con distinta composición femenina y masculina en sus Claustros, para conocer si se usa un lenguaje sexista en la redacción de sus documentos de centro. Para ello, se ha usado la Escala Val-Gen (2000), dedicada a conocer los indicadores de sexismo en los documentos de centro y en qué grado se integran los valores femeninos y de respeto a las diferencias de género, realizándose una revisión de estudios previos y comprobando qué tipo de tratamiento de género aparece recogido en los documentos de planificación educativa, centrándonos en los centros educativos seleccionados. El estudio concluye que existen todavía muchas lagunas en el tratamiento que las cuestiones de género y de igualdad de oportunidades, siendo usado un lenguaje que tiende a ser sexista y a no reconocer suficientemente la presencia de las mujeres.
\end{abstract}

Palabras clave: masculino, femenino, documentos de centro, lenguaje sexista, género

ABSTRACT: A number of linguistic mechanisms that help convey the impression that the man is the center of creativity excluding the woman. Among them is the use of generic masculine like, hiding women. Schools should keep this aspect when developing its planning documents so that both girls and boys are reflected in the daily life of schools. In this study, we selected two schools in the province of Almeria with different composition in male and female cloisters, to know if using sexist language in writing their papers center. To this end, the scale has been used Val-Gen (2000), dedicated to know the indicators of sexism in the documents of center and to what extent women are integrated values and respect for gender differences, performing a review of previous studies and checking what kind of treatment of gender appears in documents of educational planning, focusing on the selected schools. The study concludes that there are still many gaps in the treatment of gender issues and equal opportunities, being used language that tends to be sexist and does not sufficiently recognize the presence of women.

Key words: male, female, documents of center, sexist language, gender 
Vargas Muñoz, M. E. (2012). El lenguaje sexista en los documentos de planificación de los centros educativos. Espiral. Cuadernos del Profesorado, 5(9), 37-46. Disponible en: http://www.cepcuevasolula.es/espiral.

Fecha de recepción: 09/08/2011

Fecha de aceptación: 18/12/2011
Enviar correspondencia a: estelavargasmu@gmail.com

\section{1.- INTRODUCCIÓN}

Negar que en el centro educativo esté presente el sexismo por el hecho de que chicas y chicos comparten el espacio escolar es una equivocación. El currículo es una de las primeras fuentes de transmisión de estereotipos, tanto si hablamos de la planificación educativa (concretada en diversos documentos escolares), como de su desarrollo y puesta al día en el aula. De esta forma, Hargreaves (1998) opina que la influencia de las prácticas educativas y de las creencias sociales sobre masculinidad y feminidad se reflejan en la conducta del alumnado, especialmente entre los adolescentes.

Las materias escolares están inspiradas en una visión androcéntrica de las relaciones humanas. La característica del pensamiento androcéntrico es identificar lo "humano" con lo "masculino", olvidando la aportación femenina a la sociedad y subordinándola siempre a la masculina, concepto ligado al de patriarcado Lerder (1990) se refiere a este último concepto como la manifestación e institucionalización del dominio masculino sobre las mujeres y niños/as en la familia y la extensión del dominio masculino sobre las mujeres a la sociedad en general.

Desde el modelo androcéntrico se han primado los valores masculinos, asociados al mundo público (trabajo y producción de conocimientos), dotando a los valores femeninos (lo privado, lo afectivo) de una posición de segundo orden. Así, "lo privado y lo afectivo no es digno (científico) de ser transmitido en las escuelas, con lo que ignoramos las aportaciones de las mujeres en la historia y en las ciencias" (Padilla, 2002, p. 57).

Los apoyos materiales a la enseñanza tampoco son neutrales y se ha constatado la carga sexista, tanto de los tradicionales libros de texto (García et al., 1993), como de otros materiales didácticos (Espín y Rodríguez Moreno, 1996). Como esta investigación demuestra, no sólo es una cuestión de cantidad de imágenes (fotografías e ilustraciones) con presencia femenina.

También es una cuestión de cualidad/calidad de las imágenes que se presentan al alumnado, siendo especialmente importante la actividad que desempeñan los hombres y las mujeres que aparecen en ellas.

No sólo en los materiales didácticos, sino en toda situación de interacción escolar se utiliza un lenguaje sexista. Bengoechea (2003) apunta que el lenguaje refleja y, muy especialmente, ayuda a construir, nuestra concepción del mundo y la realidad.

Martínez y Alberdi (1988), recuerdan que un rápido análisis de las estructuras gramaticales nos revela que continuamente estamos utilizando mecanismos de gran sutileza que provocan la ocultación de la mujer y la masculinizando del pensamiento.

Para poder asentar las bases de este trabajo, se ha recurrido a un instrumento ya elaborado para observar cómo se recogen los valores de género en los documentos curriculares, realizado por Padilla, Moreno y Vélez (2000).

Otros estudios realizados en el contexto español, concretamente los de Espín y Rodríguez Moreno (1996) y Subirats y Tomé (1992), presentan pautas para el análisis del sexismo en los centros educativos y en ciertos tipos de materiales didácticos.

La hipótesis que se presenta para este trabajo fue la siguiente: la evolución legislativa influye en la elaboración de los documentos de los centros educativos así como en el uso de un lenguaje sexista a la hora de redactarlos. 
El objetivo principal de este estudio fue reconocer el lenguaje sexista utilizado en los documentos de centro de dos colegios de educación primaria.

\section{2.- MATERIAL Y MÉTODO}

En primer lugar se realizó una revisión de estudios previos, sacando a la luz la importancia que tiene el abordaje y la investigación sobre los métodos y procedimientos utilizados acerca del uso del lenguaje sexista en los centros educativos, continuando con una revisión bibliográfica exhaustiva y actual para conocer las diferentes investigaciones llevadas a cabo en torno a este tema.

Una vez definida la hipótesis para trabajar, los objetivos de investigación y las variables de estudio (qué investigar), se acometió el cómo hacerlo, estableciendo el procedimiento y el proceso de recogida de datos, así como los instrumentos a utilizar.

La recogida de datos, como parte fundamental de cualquier trabajo de investigación, puede realizarse con diferentes técnicas y utilizando distintos instrumentos, siempre que se asegure que tanto unos como otros sean precisos y adecuados al objeto de estudio.

Tras esto, se pasó a comprobar qué tipo de tratamiento de género aparece recogido en los documentos de planificación educativa, centrándonos en los anteriores centros educativos que han sido detallados, usando una escala de observación de variables y anotando cuáles son los indicadores del uso de un lenguaje sexista y cómo es la visión que se extrae de las mujeres. Para ello, se contabilizaron las ocasiones en las que se hacía alusión al masculino genérico así como las veces en las que se remarcaba la presencia de las mujeres en los centros elaborándose un total de nueve tablas indicando si se usaba "Nunca o casi nunca" dándole el valor 1, "algunas veces" con valor 2 y "Siempre o casi siempre" valor 3 .

Para finalizar, se introdujeron los datos recogidos en el programa SPSS con el fin de poder elaborar las tablas de resultados.

\section{Técnicas e instrumentos de recogida de información}

La elaboración del presente estudio se ha hecho atendiendo al análisis de los documentos curriculares. Para poder asentar las bases de este trabajo, se ha recurrido a un instrumento ya elaborado para observar cómo se recogen los valores de género en los documentos curriculares, realizado por Padilla, Moreno y Vélez (2000).

En él se recogen aspectos generales como son el uso de un lenguaje sexista, la presencia de las mujeres en el centro (cuántas son, qué cargos ocupan,...) además de otras cuestiones más específicas de cada tipo de documento (memorias docentes, proyecto educativo, proyectos curriculares, planes de orientación y acción tutorial).

Aunque su aplicación requiere un tiempo considerable, este instrumento presenta la ventaja de poder ser utilizado para cualquier centro educativo de enseñanza primaria y secundaria, lo que lleva a que, a través de él, se puedan realizar comparaciones entre distintos centros.

Esta escala observacional, por tanto, pretende:

- Conocer en qué grado aparecen ciertos indicadores de sexismo de carácter general (lenguaje y presencia femenina) en los documentos de centro (proyecto educativo, proyecto curricular, plan de orientación y acción tutorial y planes).

- Conocer en qué grado los objetivos, contenidos, actividades, organización y evaluación que planifica el centro escolar integran los valores femeninos y de respeto a las diferencias de género.

- Elaborar un sistema de categorías descriptivas que se recogen en una rejilla con indicadores referidos al uso del lenguaje. 


\section{Diseño}

Nos encontramos con un diseño no experimental donde el objetivo no es realizar una inferencia causal, sino meramente descriptivo, donde no existe ningún control de las variables. Por lo tanto, estamos ante un estudio descriptivo y de corte transversal (Herrera y Yshikawa, 2001).

Los estudios descriptivos son aquellos que estudian situaciones que ocurren en condiciones naturales, más que aquellos que se basan en situaciones experimentales (Merino, 2007). Permite:

- Elaborar teorías o hipótesis.

- Identificar problemas de la práctica.

- Justificar la práctica.

Es transversal ya que se lleva a cabo en un momento puntual, sin repetirse las medidas de las variables estudiadas en un periodo extendido de tiempo en diferentes ocasiones.

\section{Variables de estudio}

Las variables que se tratan a lo largo de este estudio son las siguientes:

- Uso del genérico masculino.

- Orden sistemático en la escritura.

- Conceptos, cargos, actividades que se atribuyen al género masculino.

- Conceptos, cargos, actividades que se atribuyen al género femenino.

- Uso del o/a.

- Se busca la palabra más adecuada en cada momento para evitar el o/a y recoger a ambos géneros.

- Orden variado en la escritura.

- Parrilla de análisis de elementos curriculares.

\section{Población y muestra}

La población de referencia o universo en este estudio la han conformado dos centros con características diferentes en cuanto a alumnado y profesorado.

El centro al que llamaremos centro "1" está ubicado en una localidad grande de la provincia de Almería. El profesorado lo conforman un total de 34 maestras y 12 maestros. El equipo directivo está formado por la directora y la jefa de estudios y el secretario.

El centro al que llamaremos centro "2" es un centro rural, distribuido en cinco pedanías. El profesorado lo conforman un total de 24 maestras y 6 maestros. El equipo directivo está formado por la directora y la secretaria y el jefe de estudios, siendo éste último el encargado de elaborar y coordinar los documentos de centro.

En la tabla 1 se muestran de forma más sencillas las diferencias entre el centro $1 \mathrm{y}$ centro 2.

Tras estas anotaciones, se pasó a conocer el uso del lenguaje sexista en los diferentes documentos de centro. Para ello, se utilizó la Escala Val-Gen (2000), recogida en la Tabla 9 de este estudio, gracias a la cual se pudieron anotar los indicadores referidos al uso Tabla 1.- Plantilla de los centros en función de género. del lenguaje en cada centro educativo referido.

\begin{tabular}{ccc}
\hline & CENTRO 1 & CENTRO 2 \\
\hline Maestras & 34 & 24 \\
Maestros & 12 & 6 \\
& Directora & Directora \\
Equipo directivo & Jefa de & Jefe de \\
& estudios & Estudios \\
& Secretario & Secretaria \\
\hline
\end{tabular}

\section{Análisis de datos}

Se llevó a cabo un análisis estadístico (descriptivo y transversal) de la información recabada a partir de la escala Val-Gen, cuyos datos se analizaron utilizando SPSS v.15.0. 


\section{3.- RESULTADOS}

Como se puede observar en la tabla 2, en el centro 1 se utiliza 13 veces la palabra alumno o alumnos para referirse al alumnado en general. En el centro 2, el número de veces aumenta hasta 48 . El término todos se utiliza 29 veces en el centro 2 y sólo dos veces en el centro 1.

Tabla 2. Uso del genérico masculino

\begin{tabular}{lcc}
\multicolumn{3}{c}{ Tabla 2. Uso del genérico masculino } \\
\hline INDICADORES & CENTRO 1 & CENTRO 2 \\
\hline Miembros & 6 & 4 \\
Padres & 1 & 17 \\
Hijos & 1 & 4 \\
Ellos & 2 & 9 \\
Socios & 1 & 0 \\
Disminuidos & 1 & 0 \\
Dos de ellos & 1 & 0 \\
Maestros & 4 & 0 \\
Alumno/s & 13 & 48 \\
Tutor/es & 5 & 9 \\
Niño/s & 0 & 6 \\
Los demás & 0 & 8 \\
Profesores & 0 & 12 \\
Todos & 3 & 29 \\
Distintos & 0 & 5 \\
Compañeros & 0 & 1 \\
\multicolumn{1}{c}{ MEDIA } & 2.37 & 9.5 \\
\hline
\end{tabular}

En la tabla 3, comprobamos cómo en el centro 1 se utiliza alumno y/o alumna un total de 15 veces, seguido de maestros y maestras (siete veces). En el centro 2, la expresión que más se utiliza es padres y madres (cinco veces).
Tabla 3.- Orden sistemático en la escritura.

\begin{tabular}{|c|c|c|}
\hline INDICADORES & CENTRO 1 & CENTRO 2 \\
\hline Padres y madres & 9 & 5 \\
\hline Hombres y mujeres & 1 & 0 \\
\hline Alumno o/y alumna & 15 & 0 \\
\hline Hijos e hijas & 3 & 0 \\
\hline Delegados y delegadas & 1 & 0 \\
\hline Compañeros y compañeras & 1 & 0 \\
\hline Maestros y maestras & 7 & 1 \\
\hline Tutores y tutoras & 1 & 0 \\
\hline Jefe o Jefa de estudios & 1 & 0 \\
\hline Coordinadores y coordinadoras & 1 & 0 \\
\hline Funcionarios y funcionarias & 1 & 0 \\
\hline Niños y niñas & 1 & 0 \\
\hline Profesor o profesora & 8 & 0 \\
\hline MEDIA & 3.92 & 0.46 \\
\hline
\end{tabular}

En esta tabla 4, el centro 1 se utiliza una vez el término profesor, maestro tutor y claustro de profesores respectivamente. Sin embargo, en el centro 2 se utiliza seis veces el término profesor, dos el de maestro tutor, tres veces el de director, una vez presidente y dos veces coordinador cuando estos puestos son ocupados por mujeres.
Tabla 4. Conceptos, cargos, actividades que se atribuyen al género masculino.

\begin{tabular}{|c|c|c|c|}
\hline & & CENTRO 1 & CENTRO 2 \\
\hline Profesor & & 1 & 6 \\
\hline Maestro-profesor tutor & & 1 & 2 \\
\hline Director & & 0 & 3 \\
\hline Presidente & & 0 & 1 \\
\hline Coordinador & & 0 & 2 \\
\hline Claustro de profesores & & 1 & 0 \\
\hline & MEDIA & 0,5 & 2,33 \\
\hline
\end{tabular}

En la tabla 5, observamos cómo en el centro 1, se utiliza una vez el término profesoras de apoyo de educación infantil. En el centro 2 no se atribuyen cargos al género femenino

Tabla 5.- Conceptos, cargos, actividades que se atribuyen al género femenino.

\begin{tabular}{lccc}
\hline & CENTRO 1 & CENTRO 2 \\
\hline Profesoras de apoyo de educación infantil & & 1 & 0 \\
& MEDIA & 1 & 0 \\
\hline
\end{tabular}


En esta tabla 6, en el centro 1 se usa cuatro veces el término alumnos/as o alumno/a, seguido de una respuesta para profesores/as o profesor/a. En el centro 2, el término alumnos/as o alumno/a es utilizado en 27 ocasiones, seguido de dos ocasiones para profesores/as o profesor/a $\mathrm{y}$ de tutor/a, y de una vez para compañeros/as.

Tal como podemos comprobar en la tabla 7 , en el centro 1 , el término alumnado se utiliza 83 veces, seguido de 31 ocasiones el término profesorado.

En el centro 2, el término alumnado se usa 11 veces, seguido de 10 veces el referido al profesorado.
Tabla 6.- Uso del o/a.

\begin{tabular}{|c|c|c|}
\hline & CENTRO 1 & CENTRO 2 \\
\hline Alumnos/as - Alumno/a & 4 & 27 \\
\hline Profesores/as - Profesor/a & 1 & 2 \\
\hline Niños/as & 0 & 0 \\
\hline Tutor/a & 0 & 2 \\
\hline Compañeros/as & 0 & 1 \\
\hline MEDIA & 1 & 6.4 \\
\hline
\end{tabular}

Tabla 7. Se busca la palabra más adecuada en cada momento para evitar el o/a y recoger a ambos géneros.

\begin{tabular}{|c|c|c|c|}
\hline & & CENTRO 1 & CENTRO 2 \\
\hline Alumnado & & 82 & 11 \\
\hline Familias & & 4 & 4 \\
\hline Profesorado & & 31 & 10 \\
\hline Personal & & 11 & 0 \\
\hline Secretaría & & 2 & 0 \\
\hline Comunidad escolar & & 2 & 2 \\
\hline & MEDIA & 22 & 4,5 \\
\hline
\end{tabular}

En esta tabla 8, vemos cómo en Tabla 8.- Orden variado en la escritura. el centro 1 se usa cinco veces la expresión madres y padres. En el centro

\begin{tabular}{lcc}
\hline & CENTRO 1 & CENTRO 2 \\
\hline Madres y padres & 5 & 0 \\
\hline
\end{tabular}
2 no se usa.

La tabla 9 arroja los siguientes resultados. En el centro 1, algunas veces se utiliza el masculino genérico, al igual que cargos que se atribuyen al género masculino y femenino y el uso de o/a. Siempre o casi siempre se utiliza un orden sistemático en la escritura y se busca la palabra más adecuada.

Tabla 9.- ESCALA VAL-GEN; Parrilla de análisis de elementos curriculares.

\begin{tabular}{|c|c|c|}
\hline $\begin{array}{c}\text { INDICADORES } \\
\text { Parrilla } \mathrm{n}^{\circ} 1 \text { : Indicadores referidos al uso del } \\
\text { lenguaje. }\end{array}$ & CENTRO 1 & CENTRO 2 \\
\hline Uso del masculino genérico & 2 & 3 \\
\hline Orden sistemático en la escritura & 3 & 3 \\
\hline $\begin{array}{l}\text { Conceptos cargos, actividades que se atribuyen al } \\
\text { género masculino }\end{array}$ & 2 & 3 \\
\hline $\begin{array}{l}\text { Conceptos cargos, actividades que se atribuyen al } \\
\text { género femenino }\end{array}$ & 2 & 1 \\
\hline Uso del a/o & 2 & 1 \\
\hline $\begin{array}{l}\text { Se busca la palabra más adecuada en cada momento para } \\
\text { evitar el o/a y recoger a ambos géneros }\end{array}$ & 3 & 1 \\
\hline Orden variado en la escritura & 2 & 1 \\
\hline
\end{tabular}

Nota.- 1 (Nunca o casi nunca); 2 (algunas veces); 3 (Siempre o casi siempre)

En el centro 2, nunca o casi nunca se atribuyen cargos al género femenino, no se suele utilizar o/a y no se busca la palabra más adecuada. Siempre o casi siempre se usa el masculino genérico, al igual que el orden sistemático en la escritura y los conceptos y cargos se atribuyen al género masculino.

En relación al uso del genérico: el centro 1 algunas veces utiliza el masculino genérico mientras que el centro 2 lo utiliza siempre o casi siempre (figura 1).

Orden sistemático en la escritura (figura 2): el centro 1 y el centro 2 siempre o casi siempre usan el orden sistemático en la escritura. 
Conceptos, cargos que se atribuyen al género masculino (figura 3): el centro 1 a veces atribuye al género masculino algunos conceptos o cargos. El centro 2 siempre o casi siempre atribuye estos aspectos al género masculino.

Conceptos, cargos que se atribuyen al género femenino (figura 4): el centro 1 algunas veces atribuye cargos o conceptos al género femenino. El centro 2 nunca o casi nunca.

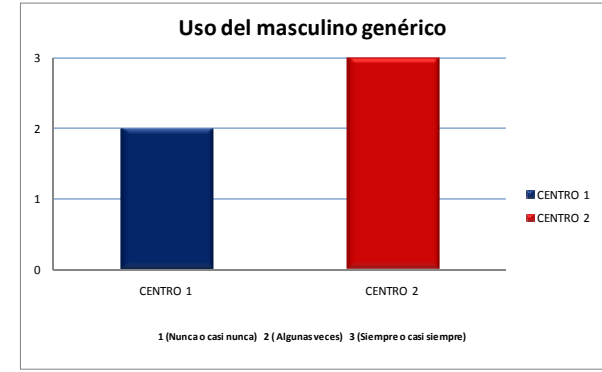

Figura 1.- Uso del masculino genérico

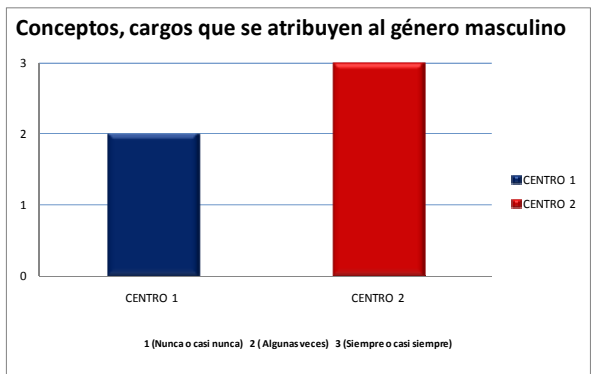

Figura 3.- Conceptos, cargos que se atribuyen al género masculino.

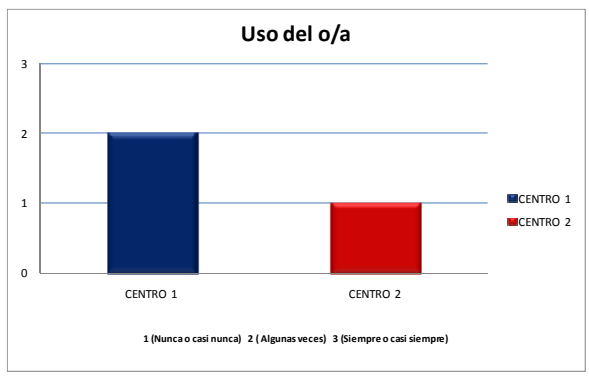

Figura 5.- Uso del o/a.

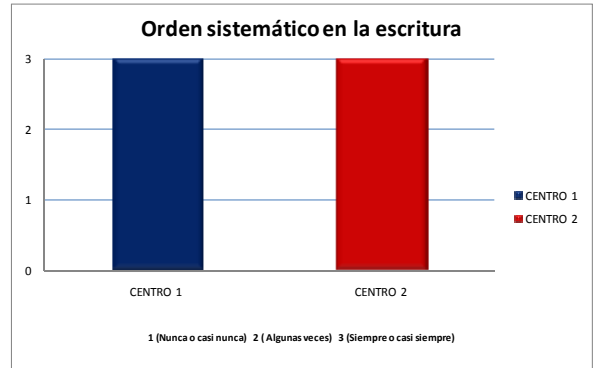

Figura 2.- Orden sistemático en la escritura

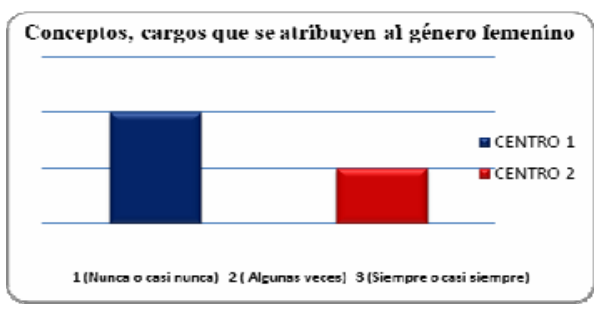

Figura 4.- Conceptos, cargos que se atribuyen al género femenino.

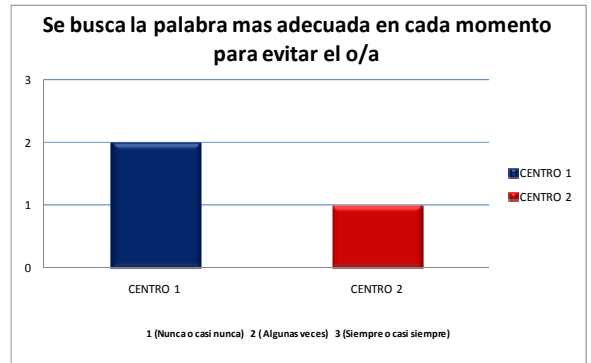

Figura 6.- Se busca la palabra más adecuada para evitar el o/a.

Uso del o/a: el centro 1 algunas veces usa o/a (figura 5). El centro 2, nuca o casi nunca lo usa.

Se busca la palabra más adecuada en cada momento para evitar el o/a (figura 6): en el centro 1 , algunas veces se busca la palabra más adecuada para evitar el o/a. en el centro 2, nunca o casi nunca se usan palabras más adecuadas que el o/a.

Como se puede comprobar, la media obtenida en el centro 1 en lo referente al uso del masculino genérico (2.37) es bastante menor que la del centro 2 (9.5), lo que significa que el primer centro intenta hacer un menor uso del masculino genérico, limitando el uso de palabras genéricas que traten de englobar a ambos géneros.

A continuación, se recogen algunas observaciones extraídas de forma literal de ambos documentos de centro donde se puede comprobar cuál es el uso del lenguaje sexista. 
- OBSERVACIONES:

CENTRO 1

“...utilizará para asambleas de sus socios dos aulas comunicadas por un panel...”

\section{CENTRO 2}

"No agredir a ningún compañero"

En cuanto al orden sistemático en la escritura, es llamativo el hecho de que en el centro 2 casi no se utilicen estas expresiones, solamente se hace en seis ocasiones. Este aspecto se debe a que no se utilizan estas estructuras, no al hecho de que se tenga en cuenta un orden en donde el género femenino sea anterior al masculino. Siempre que se mencionan a ambos géneros, se hace aludiendo primero al hombre y después a la mujer. Por ejemplo, se dice siempre los alumnos y las alumnas, los maestros y las maestras, etc. y nunca al revés, incumpliendo, además, el criterio de orden alfabético a la hora de nombrar a ambos géneros.

- OBSERVACIONES:

\section{CENTRO 2}

“Un niño o niña maltratado o acosado"

Encontramos que el centro 2 tiende a utilizar términos masculinos para referirse a los puestos de profesor, maestro, director, presidente y coordinador. Este aspecto es muy llamativo, puesto que querían referirse a cargos ocupados por mujeres.

- OBSERVACIONES:

\section{CENTRO 1}

"Otras funciones del maestro tutor en función de apoyo"

\section{CENTRO 2}

"DIRECTOR: ..."

Con respecto a cargos que se atribuyen al género femenino, en el centro 1, se destinan los cargos de apoyo y de educación infantil exclusivamente al género femenino, hecho erróneo puesto que en el centro existen dos tutores de educación infantil. Sin embargo, en el centro 2 no se atribuyen cargos al género femenino.

\section{- OBSERVACIONES:}

\section{CENTRO 1}

"Las profesoras de apoyo a Educación Infantil cubrirán las horas de dedicación..."

La media obtenida en el indicador del uso de o/a indica que en el centro 1, se limita bastante su utilización. Sin embargo, en el centro 2, se usa en múltiples ocasiones el término o/a para referirse a ambos géneros.

En el centro 1, vemos que la media de ocasiones en las que se busca la palabra más adecuada para evitar el o/a y recoger a ambos géneros es muy alta, lo que indica que este centro está más preocupado por la visibilización del género femenino en los documentos del centro. Con respecto al centro 2, comprobamos que la media es mucho más baja.

- OBSERVACIONES:

\section{CENTRO 1}

"Comisión de admisión de alumnado"

\section{CENTRO 2}

"Llevar los documentos del alumnado de su tutoría"

Sólo en el centro 1 se utiliza la expresión madres y padres, tratando así de variar el orden sistemático en la escritura. En el centro 2, no se usa esta expresión.

\section{- OBSERVACIONES:}

\section{CENTRO 1}




\section{"Madres o padres: J de la CBM e IFS"}

Con respecto a la parrilla de la escala Val-Gen, vemos que el centro 1 tiene un interés más alto en el tratamiento de género en sus documentos de centro, puesto que suele buscar la palabra más adecuada para nombrar a ambos géneros, cosa que no suele hacer el centro 2. Además, el centro 1 sí otorga cargos a las mujeres, mientras que el centro 2 no sólo no lo hace, sino que, cuando debe nombrar cargos en femenino porque son mujeres las que los ocupan, no lo hace y le otorga el cargo al género masculino.

\section{4.- CONCLUSIONES}

a) A la vista de los resultados presentados en los apartados precedentes, puede concluirse que existen todavía muchas lagunas en el tratamiento que las cuestiones de género y de igualdad de oportunidades reciben en los documentos escolares de los centros analizados.

b) Desde un plano general, el lenguaje que se usa en dichos documentos tiende a ser sexista y a no reconocer suficientemente la presencia de las mujeres.

En conjunto, la educación española ha cambiado; la escuela de ayer nada tiene que ver con la de hoy, sin duda, porque la enseñanza y el sistema educativo han experimentado transformaciones importantes en las últimas décadas. Sin embargo, a pesar de la evolución acaecida en la sociedad en los últimos tiempos, el sexismo permanece enraizado en diversas tradiciones y costumbres, que se transmiten a través de la familia, la escuela, las amistades, los medios de comunicación, etc. Más de treinta años después de la instauración de la escuela mixta, muestran que las metas fijadas no se han alcanzado y los cambios realizados han sido insuficientes. Múltiples fracturas mantienen activas diversidad de relatos culturales transmitidos y aceptados a través del sistema educativo, enseñando a los niños y a las niñas a asumir unos roles determinados. En la escuela van aprendiendo estereotipos, de manera que todas las relaciones niños/niñas y hombres/mujeres han estado marcadas por las etiquetas y sesgos de género.

Todavía las normas que tradicionalmente han regido las prescripciones de género siguen vigentes, y mantienen una división muy acusada de los comportamientos y actitudes así como de los universos sociales en los que actúan hombres y mujeres. Existen diferencias de género en las instituciones educativas, en los libros de texto, actividades, profesorado y alumnado, todos confluyen con sus imaginarios y experiencias al proceso homogeneizador de la educación.

La enseñanza recorta posibilidades, no es plural ni respeta identidades, intenta ritualizar saberes y conductas uniformando a ambos sexos. Con naturalidad se encubren unos estereotipos que discriminan, son difíciles de identificar. No se puede subestimar, a pesar de la igualdad formal, el papel crucial que desempeña la institución escolar como «perpetuadora» de las desigualdades, pues mantiene activas las diferencias de género, tratando de forma distinta a hombres y mujeres. Por esa tendencia a la reproducción sexista, es preciso incidir en el sistema educativo, es necesaria la intervención en la educación para potenciar la igualdad y romper con la tradicional discriminación de sexos, de-construir las formas sexistas del sistema educativo actual para que la escuela fomente la igualdad. En este sentido, hace falta diseñar estrategias y poner en práctica medidas concretas para eliminar el sexismo de la enseñanza y contribuir desde ella a conseguir una sociedad igualitaria. Si bien, somos conscientes de que el tratamiento de la igualdad es una iniciativa política, que depende mucho del enfoque y del valor que le otorguen los gestores públicos.

\section{5.- REFERENCIAS}

Bengoechea, M. (2003): Influencia del uso del lenguaje y los estilos comunicativos y la formación de la identidad personal. Emakunde. Gobierno Vasco. Disponible en: www.emakunde.es/images/upload/Mercedes_Bengoechea.pdf. [Consulta: 2010, 15 de mayo].

Espín, J. V., y Rodríguez Moreno, M. L. (1996): Análisis de recursos desde la perspectiva no sexista. Barcelona: Laertes.

García, M. (1993): El sexismo en los libros de texto: análisis y propuesta de un sistema de Indicadores. Madrid: Instituto de la Mujer. 
Hargreaves, A. (1998): Paradojas del cambio: la renovación de la escuela en la era postmoderna, Kikirikí, 49, 1622.

Herrera, M. E., y Yshikawa, L. E. (2001) Factores Psicosociales en los Trastornos de Adaptación en hospitalización Psiquiátrica. Lima: Perú.

Lerder, G. (1990). La creación del patriarcado. Madrid: Editorial Crítica.

Merino, T. (2007). Estudios descriptivos: tipología. Universidad Católica de Chile. Disponible en: http://escuela.med.puc.cl/Recursos/recepidem/epiDesc4.htm [Consulta: 2010, 17 de mayo]

Padilla, M. T., Moreno, E., y Vélez, E. (2000). "Diagnostico del tratamiento del género en los documentos de centro: diseño y aplicación de la escala observacional Val-Gen”. Enseñanza, 17-18, 77-98.

Padilla, M. T. (2002) Aportaciones de las estrategias observacionales al diagnóstico del sexismo en el centro educativos. $R$ XXI. Revista de Educación, 4, 299-309.

Subirats, M., y Tomé, A. (1992): Pautas de observación para el análisis del sexismo en el ámbito educativo. Barcelona: Institut de Ciencies de l'Educacio de la Universitat Autónoma. 